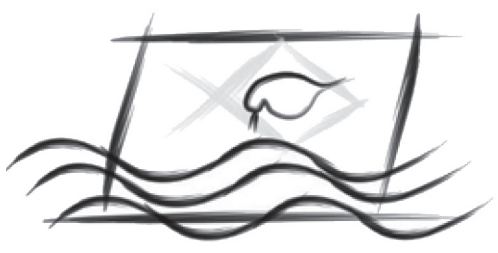

J. Braz. Soc. Ecotoxicol., v. 5, n. 1, 2010, 55-62

doi: $10.5132 /$ jbse.2010.01.009

ECOTOX - Brazil

\title{
Análise Exploratória da Incidência de Nitronaftalenos no Material Particulado Atmosférico em Área de Influência Petroquímica
}

\author{
M. A. Apel, ${ }^{1,3}$ J. A. V. Rocha, ${ }^{2}$ M. V. Coronas ${ }^{2} \&$ V. M. F. Vargas ${ }^{2,3 *}$ \\ ${ }^{1}$ Faculade de Farmácia, Universidade Federal do Rio Grande do Sul - UFRGS, Porto Alegre, Rio Grande do Sul, Brasil \\ ${ }^{2}$ Programa de Pesquisas Ambientais, Fundação Estadual de Proteção Ambiental \\ Henrique Luis Roessler - FEPAM, Porto Alegre - RS, Brasil \\ ${ }^{3}$ Curso de Especialização em Toxicologia, Pontifícia Universidade Católica do \\ Rio Grande do Sul - PUCRS, Porto Alegre - RS, Brasil
}

(Received May 5, 2008; Accepted December 23, 2009)

\begin{abstract}
RESUMO
Amostras de material particulado atmosférico, coletadas em local sob influência petroquímica, na cidade de Triunfo - Rio Grande do Sul, Brasil, foram analisadas por CG/EM-SIM para presença de nitronaftalenos. Foram analisadas amostras de Partículas Totais em Suspensão (PTS) coletadas mensalmente durante o verão, outono, inverno e primavera de 2000 e reunidas em três pools, janeiro até abril; maio até agosto; setembro até dezembro, e amostras de material particulado inalável PM10, correspondentes aos meses de fevereiro, junho e agosto de 2005. Os extratos orgânicos preparados por sonicação com diclorometano tinham sido investigados, em etapas anteriores a este estudo, quanto à presença de nitrocompostos com atividade mutagênica, pelo ensaio Salmonella/microssoma empregando linhagens sensíveis à ação dessas substâncias. A resposta mutagênica foi observada para todas as amostras em diferentes níveis mostrando que mono e dinitrocompostos estão presentes nesses extratos. Pela análise cromatográfica, objeto do presente estudo, foi verificado a predominância de dinitronaftalenos para as amostras correspondentes aos meses de inverno, tanto para a amostra de PTS, de maio a agosto, como para as amostras de PM10 relacionadas aos meses de junho e agosto. Estes resultados permitiram caracterizar de forma concordante a presença de nitronaftalenos nessas amostras com os dados obtidos pelo ensaio da atividade mutagênica.
\end{abstract}

Palavras-chave: material particulado no ar, mutagenicidade, nitronaftalenos, Salmonella/microssoma.

\section{ABSTRACT \\ Exploratory Analysis of the Incidence of Nitro-Naphthalenes in the of Airborne Particulate Matter from Petrochemical Influence Area}

Airborne particulate matter samples collected in sites under petrochemical influence, in Triunfo city - Rio Grande do Sul, Brazil, were analyzed by GC/MS-SIM for nitro-naphthalenes. Total Suspended Particulates (TSP) samples collected monthly, during summer, autumn, winter and spring of 2000 and grouped into three pools, January-April; May-August; SeptemberDecember, and three samples of particulate matter PM10, corresponding to the months of February, June and August of 2005 were analyzed. The organic extracts, obtained by sonication with dichloromethane, were previously investigated for the presence of nitrocompounds with mutagenicity activity, using Salmonella/microsome assay with sensitive strains for these substances. The mutagenic response was observed for all samples in different levels, demonstrating the presence of mono and dinitro-PAHs in the extracts. The chromatographic analysis allowed verifying the predominance of dinitronaphthalenes for the samples corresponding to the winter months, both for PTS sample, May-August, and PM10 samples, relative to June and August. These results allowed characterizing, in agreement with the mutagenic assay, the presence of nitronaphthalenes in the samples.

Keywords: airborne particulate matter, mutagenicity, nitronaphthalenes, Salmonella/microsome.

*Corresponding author: Vera Maria Ferrão Vargas, e-mail: vera.vargas@pq.cnpq.br. 


\section{INTRODUÇÃO}

Compostos mutagênicos presentes no ar urbano provêm de fontes antropogênicas, em especial fontes de combustão, e são produtos de reações químicas que ocorrem no ar, sendo que alguns destes poluentes são conhecidos por serem carcinogênicos. Tal poluição tem sido relacionada com o aumento da incidência de doenças respiratórias, como bronquite, enfisema pulmonar e câncer de pulmão, estando associada a mortes decorrentes de câncer e problemas cardiopulmonares (IARC, 1987; Lewtas; Gallagher, 1990; Gupta et al., 1996).

Muitos estudos foram conduzidos com o propósito de identificar compostos tóxicos presentes no ar e avaliar seus riscos (USEPA, 1986; Kodell et al., 1995; Chen et al., 2001, 2003; USEPA, 2003; Ciganek et al., 2004). Um dos problemas na identificação de toxicantes presentes no ar é que se trabalha com um volume muito grande e dinâmico, onde a concentração destas substâncias é muito baixa (Claxton et al., 2004). Dentre os constituintes presentes no ar foram identificados nitrohidrocarbonetos policíclicos aromáticos (nitro-HPAs), nitrilas, aminas e cetonas aromáticas (Tokiwa, 1980; Barale et al., 1994; Casellas et al., 1995; Cerna et al., 2000; Albinet et al., 2007). Estudos mostraram que compostos com 3 a 7 anéis (hidrocarbonetos policíclicos aromáticos - HPAs, oxi-HPAs, nitroarenos e anidridos aromáticos) apresentam maior efeito mutagênico. Entre os compostos identificados estão: 2-nitropireno, ciclopenta[c,d]pireno, benz[a]antraceno, benzo[a]pireno e benzo[g,h,i]pirileno (Casellas et al., 1995). Ou seja, a mutagenicidade de partículas orgânicas transportadas pelo ar é ocasionada por um grande número de compostos de várias classes químicas. Entretanto, ocorre grande disparidade entre a atividade biológica de matéria orgânica extraída de partículas do ar e a quantidade de compostos policíclicos mutagênicos e/ou carcinogênicos presentes. A maior parte da mutagenicidade é usualmente associada com classes de compostos de polaridade moderada a alta. As frações mais polares tendem a apresentar compostos nitroaromáticos, aminas aromáticas e cetonas aromáticas. A quantidade relativa de compostos e classes químicas específicas é dependente das fontes de ar, do tamanho da partícula coletada (quanto menor, maior o efeito mutagênico) e das condições meteorológicas.

Hidrocarbonetos policíclicos aromáticos (HPAs) são compostos ubíquos, formados em processos de combustão incompleta de matéria orgânica. São substâncias planas formadas por carbono e hidrogênio. O interesse no estudo da contaminação ambiental por estas substâncias está diretamente relacionado com seus efeitos biológicos, pois vários componentes deste grupo apresentam efeitos carcinogênicos e mutagênicos (Durant et al., 1996, 1999; Ciganek et al., 2004; Albinet et al., 2007).

Naftalenos e seus alquil derivados (metil-naftalenos - MNs, etil-naftalenos - ENs, e dimetil-naftalenos - DMNs) são HPAs semivoláteis presentes na atmosfera, predominantemente na fase gasosa (Atkinson, 2000; Reisen et al., 2003) e contribuem com a mutagenicidade observada na fase de vapor em amostras de ar (Harger et al., 1992; Gupta et al., 1996). Reações de combustão e atmosféricas de dióxido de nitrogênio com estes hidrocarbonetos resultam em um grande número de nitrocompostos (Dimashki et al., 2000; Phousongphouang; Arey, 2003a, b). Os produtos de reações atmosféricas dos HPAs incluem derivados nitrados mutagênicos (Arey et al., 1992; Cecinato et al., 2001; Feilberg et al., 2002; Reisen et al., 2003; Fanucchi et al., 2004).

A contaminação atmosférica é uma das maiores preocupações a cerca da poluição ambiental, afetando a qualidade de vida e a saúde humana (Vargas et al., 1998). Estudos com extratos orgânicos de material particulado, realizados nas cidades do Rio de Janeiro, São Paulo e Porto Alegre, confirmam a mutagenicidade relacionada a atividades industriais e emissões a partir de veículos (Miguel et al., 1990; Vargas et al., 1993; Sato et al., 1995; Ducatti; Vargas, 2003).

Considerando o exposto e a alta incidência de nitronaftalenos no ar, este trabalho tem como objetivo a investigação exploratória da presença de mononitro e dinitronaftalenos por cromatografia a gás acoplada a espectrometria de massas (CG-EM) em amostras de material particulado atmosférico, coletadas em local sob influência petroquímica, na cidade de Triunfo - RS, Brasil.

\section{MATERIAL E MÉTODOS}

\section{Coleta das amostras}

O local de amostragem (29 49'35" S e $\left.51^{\circ} 24^{\prime} 56^{\prime \prime} \mathrm{W}\right)$ estava situado em área sob influência petroquímica, na cidade de Triunfo - Rio Grande do Sul, Brasil. A estação de amostragem foi colocada a $6,1 \mathrm{~km}$ de distância da chaminé da central de matérias-primas desse complexo industrial petroquímico, no quadrante principal de dispersão atmosférica desse complexo e a $1,4 \mathrm{~km}$ de uma rodovia federal. Esse complexo (14,600 ha) está situado em uma região com influência mista urbana, rural e industrial na bacia hidrográfica do Caí, $30 \mathrm{~km}$ à montante da cidade de Porto Alegre, capital do estado. As amostras de partículas totais em suspensão (PTS, partículas $<100 \mu \mathrm{m}$ ), (A), (B) e (C), foram coletadas mensalmente durante o verão, outono, inverno e primavera de 2000 e reunidas em três pools (A), janeiro até abril; (B) maio até agosto; (C) setembro até dezembro. As amostras de material particulado inalável PM10 (partículas $<10 \mu \mathrm{m}$ ), (D), (E) e (F), correspondem a pools de 4 (D), 5 (E) e 5 filtros (F), relativos aos meses de fevereiro, junho e agosto de 2005.

As amostras de PTS foram coletadas em filtros de fibra de vidro (AP 40-810, 20x25 cm Millipore) utilizando um amostrador de grandes volumes de ar (General Metal Works Inc.) operando a um fluxo de 1,3-1,5 $\mathrm{m}^{3} \cdot \mathrm{min}^{-1}$ por 24 horas. As partículas MP10 (PM 10) em filtros (TX40HI20WW, $254 \times 203 \mathrm{~mm}$ ) utilizando um amostrador de grandes volumes de ar para partículas de até $10 \mu \mathrm{M}$, AGV MP10, modelo 1200/CVV, por período de 24 horas. Os filtros foram pesados e estabilizados antes e após a amostragem ( $45 \%$ de umidade). As partículas PTS e as partículas de MP 10 foram expressas em unidades $\mu \mathrm{g} . \mathrm{m}^{-3}$ de amostra de ar (ABNT, 1988). Todas as amostras foram armazenadas em freezer a $-80^{\circ} \mathrm{C}$ até o momento das análises. 


\section{Preparação das amostras}

Os filtros contendo material particulado foram submetidos à extração pela técnica de ultra-som, que consiste na obtenção de extratos de frações com afinidade diferenciada pelo solvente diclorometano (Vargas et al., 1998).

\section{Análise por cromatografia a gás acoplada a espectrometria de massas (CG/EM)}

As amostras foram analisadas em um cromatógrafo gasoso Shimadzu QP5000 com quadrupolo cilíndrico, operando com energia de ionização de $70 \mathrm{eV}$. Foi utilizada coluna capilar de sílica fundida $\mathrm{DB}_{5}(25 \mathrm{~m} \times 0,25 \mathrm{~mm} \times 0,25 \mu \mathrm{m})$ para a separação dos constituintes e hélio como gás de arraste. $\mathrm{O}$ injetor foi operado a $290{ }^{\circ} \mathrm{C}$ (modo splitless) e detector a $280{ }^{\circ} \mathrm{C}$. A seguinte programação da temperatura foi utilizada: temperatura inicial de $80^{\circ} \mathrm{C}$ (mantida por 3 minutos) seguido de aumento de $40{ }^{\circ} \mathrm{C} \cdot \mathrm{min}^{-1}$ até $220^{\circ} \mathrm{C}$ (mantida por 2 minutos), e posterior aumento da temperatura de $40^{\circ} \mathrm{C} \cdot \mathrm{min}^{-1}$ até $280{ }^{\circ} \mathrm{C}$ (mantida por 2 minutos) e de $40{ }^{\circ} \mathrm{C} \cdot \mathrm{min}^{-1}$ até $300^{\circ} \mathrm{C}$, permanecendo por 2,5 minutos. O espectrômetro de massas foi operado no modo SIM (Monitoramento Seletivo de Íons). Foram selecionados a partir da literatura especializada e espectroteca NIST62 os íons m/z 218, 172, 142, 126 e 114, para caracterizar os dinitronaftalenos, e os íons m/z 201, 156, 129 e 115 para os mononitronaftalenos (Bastow et al., 2000; Phousongphouang; Arey, 2003a; Reisen et al., 2003).

\section{RESULTADOS E DISCUSSÃO}

\section{Análise química por CG/EM-SIM}

Naftalenos e seus alquil e nitro derivados são HPAs semivoláteis presentes no ar, predominantemente na fase gasosa, sendo, geralmente, os HPAs de maior ocorrência no ar atmosférico (Ciganek, 2004; Albinet et al., 2007). A Figura 1 apresenta o perfil cromatográfico das amostras analisadas por CG/ EM-SIM, onde os íons 201, 156, 129 e 115 foram monitorados, a fim de caracterizar a presença de mononitronaftalenos. É possível verificar certa semelhança entre os cromatogramas das amostras de (A), (C), (D) e (E), podendo ser observado picos com o mesmo tempo de retenção e, para alguns, um padrão de fragmentação similar, como, por exemplo, para o pico 3 das coletas (C), (D) (E) e o pico 4 de (A) (Figura 2). Para a coleta (F), o cromatograma revelou um perfil distinto, com um pico predominante. A amostra (B) apresentou baixa ocorrência de mononitronaftalenos. Analisando o fragmentograma obtido para estas amostras, o íon 156 representa a perda do grupamento $\mathrm{NO}_{2}$ e o íon 129 a perda de duas metilas, sugerindo tratar-se de dimetilnitronaftalenos (DMNNs) e/ou etilnitronaftalenos (ENNs). Entretanto, para correta identificação dos constituintes da amostra é necessário uso de padrões.(Figuras 1 e 2)

O perfil cromatográfico em relação aos dinitronaftalenos está apresentado na Figura 3. Para este grupo de compostos foram monitorados os íons 218, 172, 142, 126, 114, para caracterizar a presença de dinitronaftalenos. É interessante observar a semelhança cromatográfica entre as amostras de (D) e (A), onde é verificada a presença de um pico predominante com o mesmo tempo de retenção em espectro de massas (Figura 4). Pela análise do fragmentograma destas duas amostras, o íon 126 corresponde à perda de duas moléculas de $\mathrm{NO}_{2}$, o íon 172 à perda de uma molécula de $\mathrm{NO}_{2}$ e o íon 142 representa a perda de uma molécula de $\mathrm{NO}_{2}$, mas aponta para possível presença de um grupamento metila, sugerindo que o composto possa ser um metil-dinitronaftaleno. Para as amostras (B) e (E) é observada uma grande incidência de compostos. Para a primeira, com exceção do pico 9, observa-se um padrão nos espectros de massas dos diversos picos, com mínimas variações de intensidade dos íons. A Figura 5 apresenta o fragmentograma do pico 9 da primeira amostra e um espectro de massas representativo, relacionado aos demais picos, levando em consideração a semelhança dos mesmos para estas duas amostras. Para a segunda, o mesmo é observado, porém todos os picos apresentam espectro de massas com o mesmo padrão daquele apresentado para a maioria dos picos na amostra de (B). Pequenas variações na intensidade são verificadas, mas sem alterar seu perfil.

Da mesma forma que para os mononitronaftalenos, a amostra (F) apresenta um perfil singular, com duas substâncias principais (Figura 4). Para (C) é verificada baixa incidência de dinitronaftalenos. Pelos resultados obtidos por CG/EM-SIM é possível inferir que as amostras (A), (C) e (D) apresentam maior incidência de mononitronaftalenos. Para as coletas (E) e (B) é observado o oposto, ou seja, maior ocorrência de dinitronaftalenos. A amostra (F) apresentou um comportamento distinto com número de picos equilibrados de mononitro e dinitronaftalenos.

Os resultados observados neste trabalho corroboram com aqueles encontrados em outros estudos do ar atmosférico em regiões próximas de zona industrial e em áreas urbanas com grande emissão de poluentes. Nestas regiões, mono e dinitronaftalenos foram os derivados de nitro-HPAs mais abundantes presentes na fase de vapor (Phousongphouang; Arey, 2003b; Ciganek et al., 2004; Albinet et al., 2007) e um dos responsáveis pelo efeito mutagênico observado para os compostos presentes no ar.

\section{Avaliação mutagênica e citotóxica: ensaio Salmonella/microssoma}

Os extratos foram previamente testados para mutagenicidade (Coronas et al., 2008) pelo ensaio Salmonella/microssoma (Maron; Ames, 1983), pelo método de microssuspensão (Kado et al., 1986). Esta modificação do ensaio permite avaliar pequenas quantidades de amostras, apresentando uma sensibilidade aproximadamente dez vezes maior em relação à metodologia clássica. Os extratos orgânicos foram avaliados inicialmente para danos do tipo erro no quadro de leitura (linhagem TA98), sendo as amostras positivas diagnosticadas quanto à presença de nitroderivados através de linhagens sensíveis a nitrocompostos formados pelas redutases bacterianas. Os extratos orgânicos obtidos a partir de PTS, no período de 2000, foram testados com o conjunto de linhagens nitrodeficientes (TA98NR, deficiente na nitroredutase clássica; TA98/1,8DNP ${ }_{6}$ deficiente em O-acetiltransferase) (Rosenkranz, 1996). Os extratos obtidos 

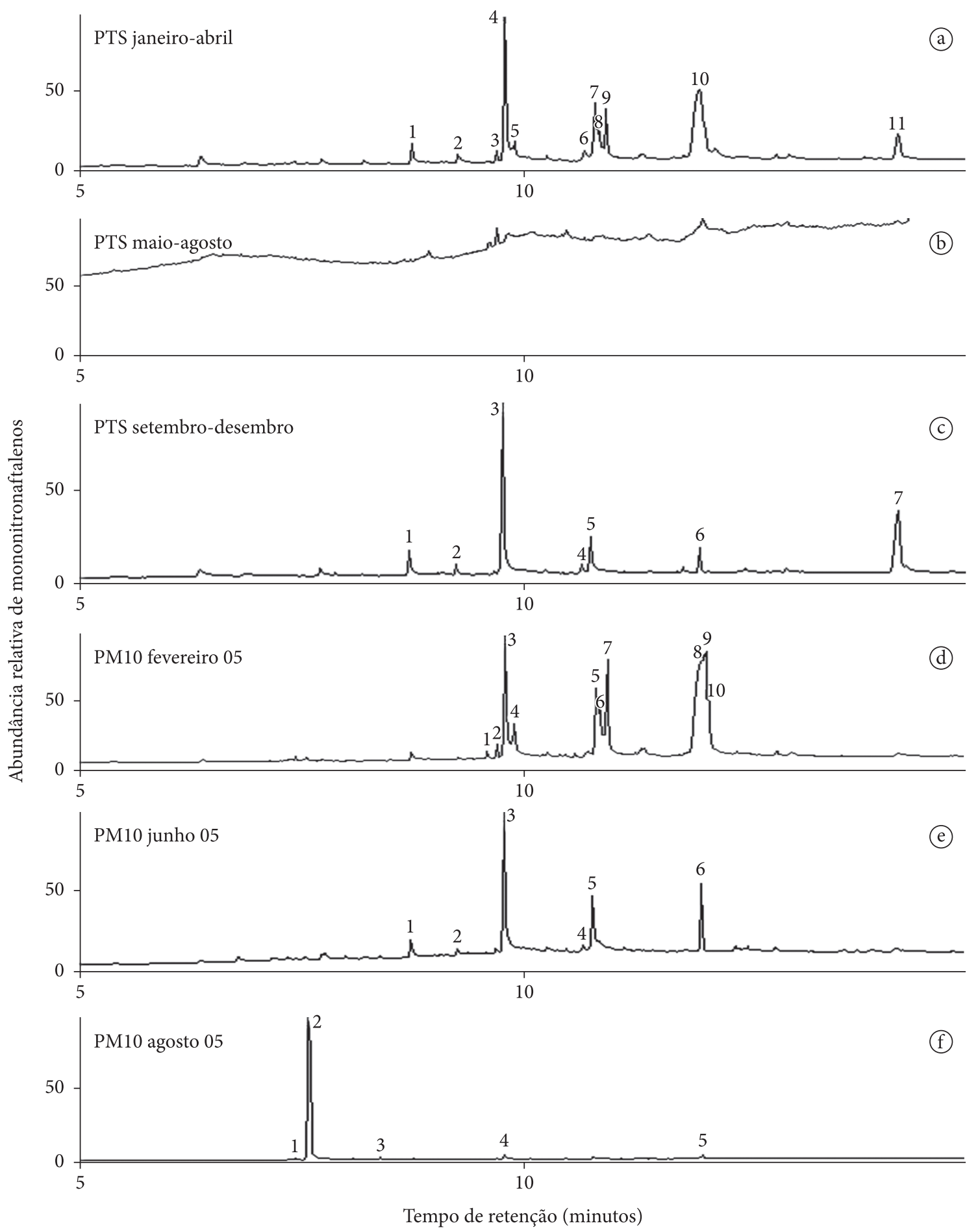

Figura 1 - Perfil cromatográfico obtido por CG/EM-SIM, em coluna $\mathrm{DB}_{5}$, para os mononitronaftalenos (m/z 201, 156, 129, 115) para as amostras (a), (b), (c), (d), (e) e (f).

a partir de PM10 foram avaliados pelo conjunto de linhagens YGs com propriedades semelhantes às nitrodeficientes, mas apresentando alta produção dessas enzimas (YG1021, que possui inserido no plasmídio pYG216 o gene da nitroredutase clássica; YG1024, que possui inserido no plasmídio pYG219 o gene da O-acetiltransferase) e, consequentemente, a maior sensibilidade para nitrocompostos, como nitroarenos (pYG216) ou aminas aromáticas (pYG219) (Watanabe et al., 1989; 1990; Umbuzeiro; Vargas, 2003).
As curvas doses-resposta, obtidas nos diferentes ensaios, foram avaliadas pelo programa Salmonel (Myers et al., 1991) conforme descrito por Vargas et al. (1993) selecionando os modelos linear e Bernstein. As amostras positivas foram expressas em revertentes $h$ is $+/ \mu \mathrm{g}$ do extrato avaliado.

A partir dessa análise foi possível detectar na amostra (A) e (B) uma mistura de mononitro e dinitrocompostos, com leve predominância de dinitrocompostos para (A), explicando a atividade mutagênica observada. Já para $(\mathrm{C})$ a sensibilidade 
frente à linhagem TA98NR indica a predominância de mononitrocompostos explicando a ação desse extrato. Nas amostras de 2005, a presença de mononitro e dinitrocompostos foi observada nos três períodos analisados, sendo que para (D) e (E) foi identificada uma contribuição maior no efeito de dinitrocompostos (maior sensibilidade frente à linhagem YG1024), e para (F) uma mistura destes dois grupos de compostos.

Conforme a sensibilidade apresentada para as linhagens nitrosensíveis no ensaio Salmonella/microssoma, é possível inferir se a mutagenicidade observada pode ser atribuída principalmente para mononitro ou dinitrocompostos. Neste caso, se a sensibilidade for maior para as linhagens TA98NR ou YG1021 indica que a ação está relacionada principalmente com a presença de mononitrocompostos. No entanto, se a sensibilidade

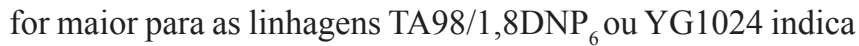
relação principalmente com presença de dinitrocompostos. Já se a resposta apresenta valores semelhantes para as linhagens empregadas (TA98NR e TA98/1, 8DNP 6 ou YG1021 e YG1024) significa uma contribuição equilibrada entre mononitro- e dinitrocompostos para o efeito. É importante salientar que este ensaio relaciona a ação apenas com nitrocompostos de forma geral, não sendo específico para nitronaftalenos ou outro grupo químico em especial.

Comparando os resultados obtidos por CG/EM-SIM (Tabela 1) com os obtidos no ensaio Salmonella/microssoma, é possível verificar certa compatibilidade dos achados para as amostras (C), (E) e (F), onde é verificado que o efeito para

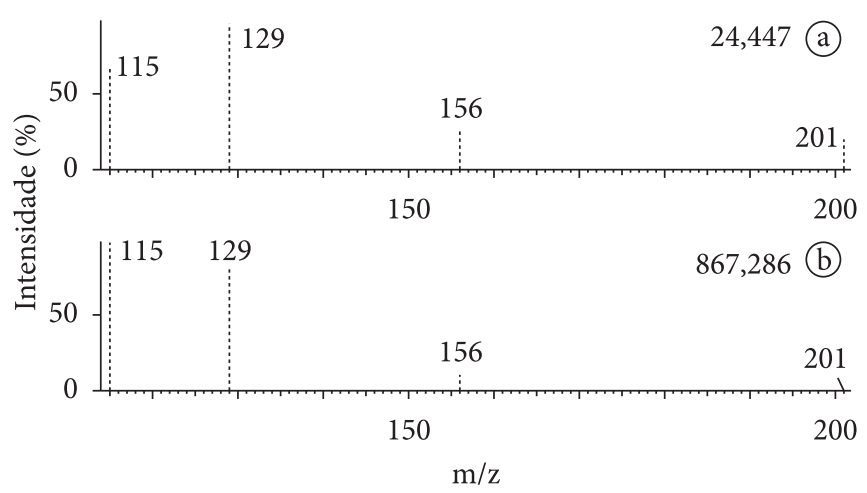

Figura 2 - Fragmentograma obtido por CG/EM-SIM, em coluna $\mathrm{DB}_{5}$, do principal composto (mononitronaftaleno) observado para as amostras de (A), (C), (D) e (E), apresentando mesmo tempo de retenção e espectro de massas (a); e do composto majoritário da coleta (F) (b).
(C) pode estar relacionado com a presença, em especial, de mononitronaftalenos, tendo em vista a queda total no efeito mutagênico sobre a linhagem TA98NR. Para a amostra (E) a atividade mutagênica demonstrou estar relacionada em particular com a presença de dinitrocompostos, confirmada pelas análises por CG/EM-SIM; e para a amostra (F) o efeito está relacionado tanto a mononitro quanto a dinitrocompostos, ocorrendo um equilíbrio entre os resultados. Pela análise por CG/EM-SIM pode ser notado também um equilíbrio nos resultados, sem maior incidência aparente de um ou outro grupo de nitronaftalenos.

Para os demais extratos podem ser feitas algumas considerações. A amostra (D) apresenta um efeito mutagênico maior frente à linhagem YG1024, indicando principalmente a presença de dinitrocompostos. Entretanto, pela análise cromatográfica, mononitronaftalenos apresentam, aparentemente, maior número de picos que dinitronaftalenos. O efeito mutagênico observado pode ser atribuído à presença de poucos compostos, porém com ação pronunciada. O mesmo pode ser dito para a amostra (A) a qual apresenta leve predominância de dinitrocompostos. Para a amostra (B), onde a atividade biológica é atribuída aos dois grupos (mononitro e dinitrocompostos) de forma equilibrada, a análise química revelou predominância de dinitronaftalenos. Esse efeito mutagênico de similar intensidade pode ser atribuído à presença de mononitrocompostos tão reativos quanto os dinitrocompostos, mesmo em baixas concentrações.

É importante ainda ressaltar a similaridade entre os cromatogramas das amostras (B) e (E) quanto a dinitronaftalenos. No entanto, as respostas com os biomarcadores de mutagenicidade mostram ausência de sensibilidade frente à linhagem TA98/1,8DNP 6 para (B) e presença de resposta frente a YG1024 para a amostra (E). Convém mencionar que a linhagem YG também é sensível para aminas aromáticas, contribuindo para a ação observada, o que não ocorre com a nitrodeficiente (TA98/1,8DNP ${ }_{6}$ ). No entanto, aminas aromáticas não apresentam o mesmo padrão de fragmentação observado para nitronaftalenos, ou seja, mesmo com ausência de padrões para comprovar a identidade dos picos encontrados, estes não se tratam de aminas aromáticas. De qualquer forma, não é possível excluir a participação destas nos efeitos observados, o que explicaria as diferenças de sensibilidade biológica detectadas. Novas análises químicas devem ser realizadas com o objetivo de verificar a possível presença dessa classe.

Tabela 1 - Comparação do efeito mutagênico observado para as amostras pelo ensaio da Salmonella/microssoma com a caracterização por CG/EM-SIM.

\begin{tabular}{|c|c|c|c|c|}
\hline \multirow[t]{2}{*}{ Amostras } & \multicolumn{2}{|c|}{ Ensaio Salmonella/microssoma ${ }^{1}$} & \multicolumn{2}{|c|}{ CG/EM-SIM } \\
\hline & Nitrocompostos & Dinitrocompostos & Mononitronaftalenos & Dinitronaftalenos \\
\hline A & + & +0 & $* *$ & * \\
\hline B & + & + & * & $* *$ \\
\hline $\mathrm{C}$ & ++ & & $* *$ & * \\
\hline $\mathrm{D}$ & + & ++ & $* *$ & * \\
\hline $\mathrm{E}$ & + & ++ & * & $* *$ \\
\hline $\mathrm{F}$ & + & + & $* *$ & $* *$ \\
\hline
\end{tabular}

${ }^{1}$ Dados apresentados em Coronas et al., 2008: +: contribuição para o efeito mutagênico; +o, ++: contribuição leve ou acentuada para o efeito mutagênico;

*: presença do grupo químico; **: presença predominante do grupo químico. 


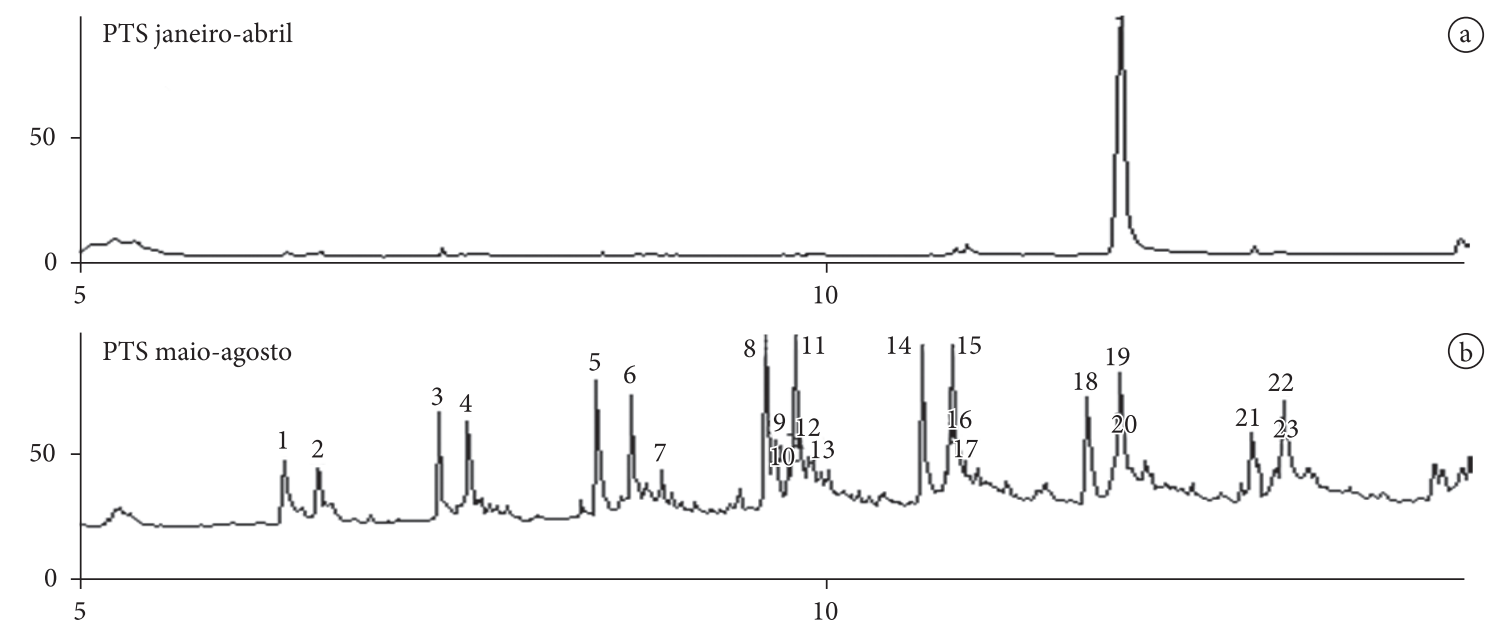

(a)

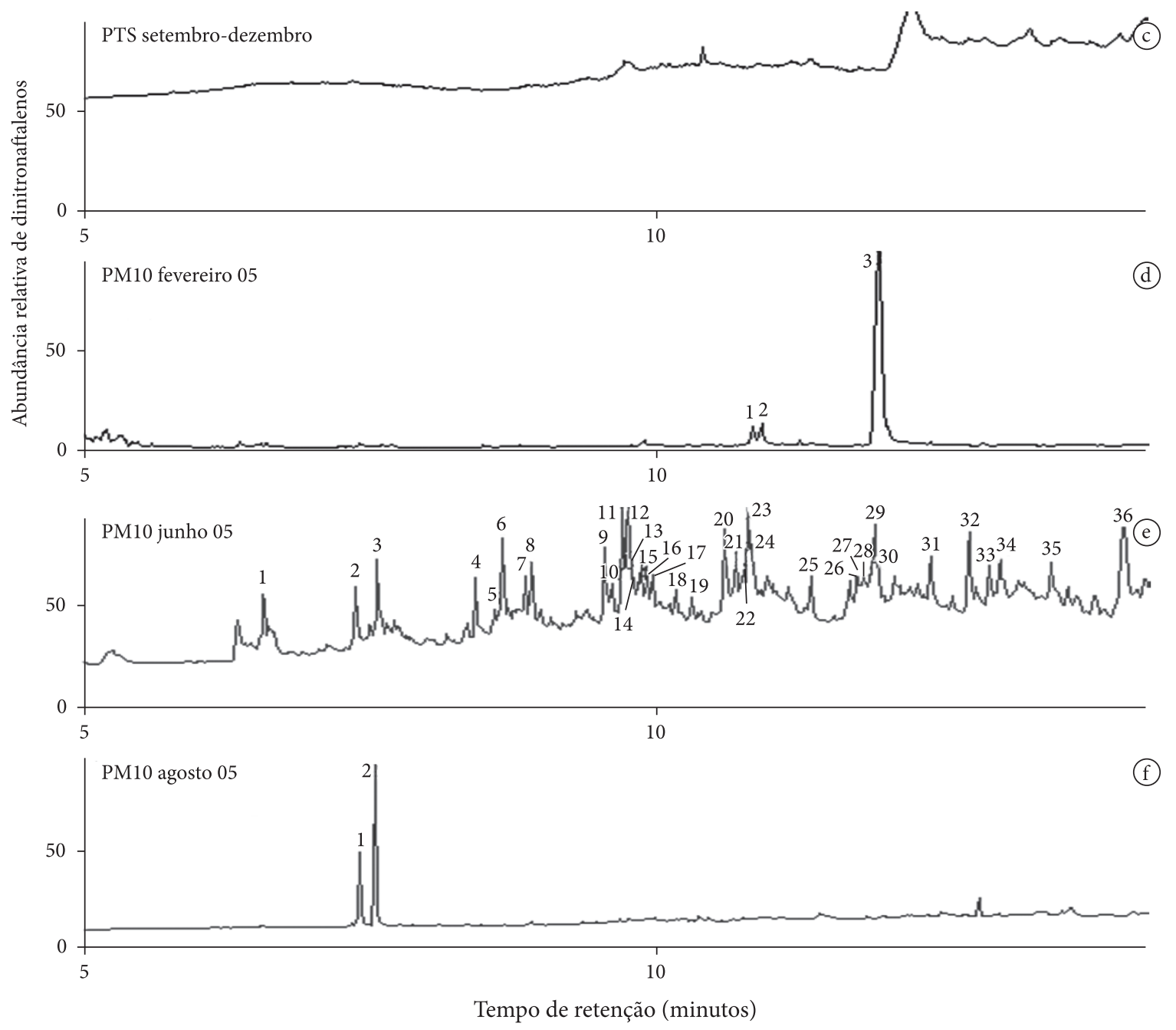

Figura 3 - Perfil cromatográfico obtido por CG/EM-SIM, em coluna DB5, para os dinitronaftalenos (m/z 218, 172, 142, 126, 114) para as amostras (a), (b), (c), (d), (e) e (f).

Podemos concluir que, de modo geral, houve uma similaridade entre os resultados obtidos no diagnóstico da presença de mononitro e dinitrocompostos, utilizando biomarcadores biológicos para mutagênese, e as análises químicas especializadas. No entanto, a definição dos compostos responsáveis pela reatividade mutagênica somente será possível através da comparação com padrões específicos. A etapa de trabalho já realizada fornece uma base exploratória de algumas possíveis classes químicas responsáveis pela resposta mutagênica.

Deve, ainda, ser considerado que o efeito biológico mede a atividade da mistura de compostos presentes no extrato total e não apenas a ação de uma classe específica. No entanto, são bem estabelecidas as vantagens em utilizar o fracionamento bioguiado como uma estratégia metodológica com o objetivo 


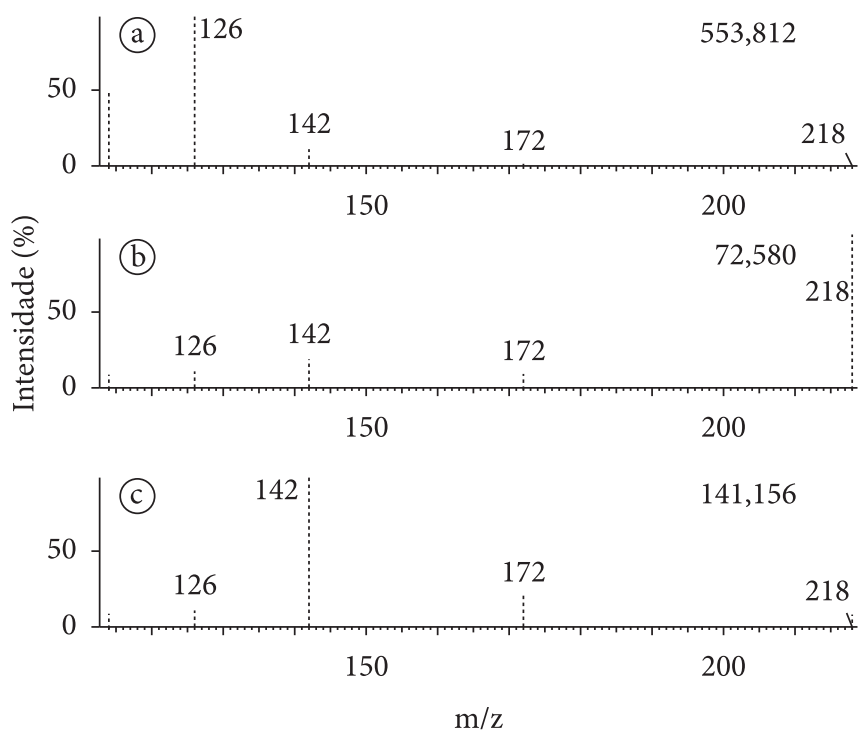

Figura 4 - Fragmentograma obtido por CG/EM-SIM, em coluna $\mathrm{DB}_{5}$, para os dinitronaftalenos $(\mathrm{m} / \mathrm{z} 218,172,142,126,114)$ do principal composto observado para as amostras de (a) e (d), apresentando mesmo tempo de retenção e espectro de massas (a); e dos dois compostos predominantes da coleta de (f) (b e c).

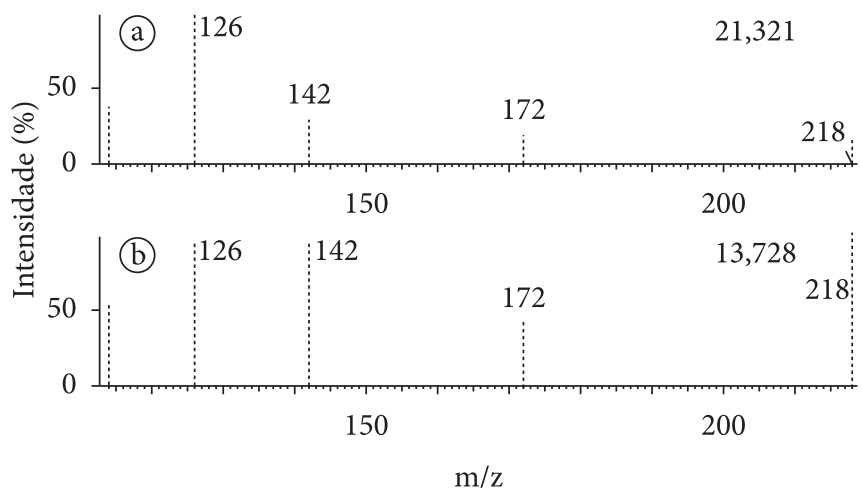

Figura 5 - Fragmentograma representativo, obtido por CG/EM-SIM em coluna $\mathrm{DB}_{5}$ para os dinitronaftalenos (m/z 218, 172, 142, 126, 114), relacionado aos diversos picos da amostra de (b) e (e), apresentando padrão de fragmentação similar (a); e do pico 9 da amostra (b).

de definir a substância ou o grupo de compostos responsáveis pela ação mutagênica (Brack et al., 1999; Reineke et al., 2002; Vargas, 2003; Claxton et al., 2004). Desta forma, este trabalho foi delineado no sentido de verificar os constituintes presentes no extrato diclorometano, uma vez que neste solvente encontram-se muitos compostos com reconhecida atividade mutagênica, como é o caso dos nitronaftalenos aqui estudados. Investigações futuras serão conduzidas com o objetivo de identificar as substâncias responsáveis pelos efeitos observados, além de explorar novas classes químicas que podem estar presentes neste material de partida, contribuindo com a ação.

Agradecimentos - Os autores agradecem as atividades prévias a este estudo realizadas pela Bióloga Adriana Ducatti na realização dos ensaios para avaliar a mutagenicidade dos extratos e o diagnóstico da presença de mononitro e dinitrocompostos através das linhagens nitrosensíveis. Este trabalho contou com o auxílio financeiro CNPq e CAPES.

\section{REFERÊNCIAS BIBLIOGRÁFICAS}

ABNT, Associação Brasileira de Normas Técnicas, 1988, NBR 9547 - Material particulado em suspensão no ar ambiente. Determinação da concentração total pelo método do amostrador de grande volume. ABNT, Rio de Janeiro.

ALBINET, A., LEOZ-GARZIANDIA, E., BUDZINSKI, H. \& VIILENA, V. E. E., 2007, Polycyclic aromatic hydrocarbons (PAHs), nitrated PAHs and oxygenated PAHs in ambient air of the Marseilles area (South of France): Concentrations and sources. Sci. Total Environ., 384: 280-292. doi:10.1016/j. scitotenv.2007.04.028.

AREY, J., HARGER, W. P., HELMIG, D. \& ATKINSON, R., 1992, Bioassay-directed fractionation of mutagenic PAH atmospheric photooxidation products and ambient particulate extracts. Mutat. Res., 281: 67-76. doi:10.1016/0165-7992(92)90038-J.

ATKINSON, R., 2000, Atmospheric chemistry of VOCs and NOx. Atmos. Environ., 34: 2063-2101. doi:10.1016/S13522310(99)00460-4.

BARALE, R., GIROMINI, L., DEL, R. Y. S., BARNINI, B., BULLERI, M., BARRAI, I., VALERIO, F., PALA, M. \& HE, J., 1994, Chemical and mutagenic patterns of airborne particulate matter collected in 17 Italian towns. Environ. Health Perspect., 102: $67-73$.

BASTOW, T. P., ALEXANDER, R., FISHER, S. J., SINGH, R. K., VAN AARSSEN, B. G. K. \& KAGI, R. I., 2000, Geosynthesis of organic compounds. Part V-methylation of alkylnaphthalenes. Org. Geochem., 31: 523-534. doi:10.1016/S0146-6380(00)00038-3.

BRACK, W., ALTENBURGER, R., ENSENBACH, U., MÖDER, M., SEGNER, H. \& SCHÜÜRMANN, G., 1999, BioassayDirected Identification of Organic Toxicants in River Sediment in the Industrial Region of Bitterfeld (Germany) - A Contribution to Hazard Assessment. Arch. Environ. Contam. Toxicol., 37: 164174. doi: $10.1007 / \mathrm{s} 002449900502$.

CASELLAS, M., FERNANDEZ, P., BAYONA, J. M. \& SOLANAS, A. M., 1995, Bioassay-directed chemical analysis of genotoxic components in urban airborne particulate matter from Barcelona (Spain). Chemosphere, 30: 725-740. doi:10.1016/00456535(94)00438-Z.

CECINATO, A., MABILIA, R., BRACHETTI, A., DI FILIPPO, P. \& LIBERTI, A., 2001, Nitrated-PAH in urban air of Italy as indicators of motor vehicle emission and light-induced reactions. Anal. Lett., 34: 927-936. doi: 10.1081/AL-100103603.

CERNA, M., POCHMANOVA, D., PASTORKOVA, A., BENES, I., LENICEK, J., TOPINKA, J. \& BINKOVA, B., 2000, Genotoxicity of urban air pollutants in the Czech Republic. Part I. Bacterial mutagenic potencies of organic compounds adsorbed on PM10 particulates. Mutat. Res., 469: 71-82. doi:10.1016/ S1383-5718(00)00062-0.

CHEN, J. J., CHEN, Y. J., RICE, G., TEUSCHLER, L. K., HAMERNIK, K., PROTZEL, A. \& KODELL, R. L., 2001, Using dose addition to estimate cumulative risks from exposures to multiple chemicals. Regul. Toxicol. Pharmacol., 34: 35-41. doi:10.1006/rtph.2001.1485.

CHEN, J. J., CHEN, Y. K., TEUSCHLER, L. K., RICE, G., HAMERNIK, K., PROTZEL, A. \& KODEL, R. L., 2003, Cumulative risk assessment for quantitative response data. Environmetrics., 14: 339-353. doi: 10.1002/env.587.

CIGANEK, M., NECA, J., ADAMEC, V., JANOSEK, J. \& MACHALA, M., 2004, A combined chemical and bioassay analysis of traffic-emitted polycyclic aromatic hydrocarbons. Sci. Total Environ., 334-335: 141-148. doi:10.1016/j. scitotenv.2004.04.034.

CLAXTON, L. D., MATTHEWS, P. P. \& WARREN, S. H., 2004, The genotoxicity of ambient outdoor air, a review: Salmonella 
mutagenicity. Mutat. Res., 567: 347-399. doi:10.1016/j. mrrev.2004.08.002.

CORONAS, M. V., HORN, R. C., DUCATTI, A.; ROCHA, J. V. \& VARGAS, V. M. F., 2008, Mutagenic activity of airborne particulate matter in a petrochemical industrial area. Mutat. Res., 650: 196-201. doi:10.1016/j.mrgentox.2007.12.002.

DIMASHKI, M., HARRAD, S. \& HARRISON, R. M., 2000, Measurements of nitro-PAH in the atmospheres of two cities. Atmos. Environ., 34: 2459-2469. doi:10.1016/S13522310(99)00417-3

DUCATTI, A. \& VARGAS, V. M. F., 2003, Mutagenic activity of airborne particulate matter as an indicative measure of atmospheric pollution. Mutat Res., 540: 67-77. doi:10.1016/ S1383-5718(03)00170-0.

DURANT, J. L., BUSBY Jr., W. F., LAFLEUR, A. L., PENMAN, B. W. \& CRESPI, C. L., 1996, Human cell mutagenicity of oxygenated, nitrated and unsubstituted poycyclic aromatic hydrocarbons associated with urban aerosols. Mutat. Res., 371: 123-157. doi:10.1016/S0165-1218(96)90103-2.

DURANT, J.L.,LAFLEUR,A.L., BUSBYJr., W.F., DONHOFFNER, L. L., PENMAN, B. W. \& CRESPI, C. L., 1999, Mutagenicity of C24H14 PAH in human cell expressing CYP1A1. Mutat. Res., 446: 1-14. doi:10.1016/S1383-5718(99)00135-7.

FANUCCHI, M. V., KIMBERLY, C. D., CLAY, C. C. \& PLOPPER, C. G., 2004, Increased vulnerability of neonatal rats and mice to 1-nitronaphthalene-induced pulmonary injury. Toxicol. Appl. Pharmacol., 201: 53-65. doi:10.1016/j.taap.2004.05.002.

FEILBERG, A., NIELSEN, T., BINDERUP, M. L., SKOV, H. \& POULSEN, M. W. B., 2002, Observations of the effect of atmospheric processes on the genotoxic potency of airborne particulate matter. Atmos. Environ., 36: 4617-4625. doi:10.1016/ S1352-2310(02)00462-4.

GUPTA, P., HARGER, W. P. \& AREY, J., 1996, The contribution of nitro- and methylnitro-naphthalenes to the vapor-phase mutagenicity of ambient air samples. Atmos. Environ., 30: $3157-$ 3166. doi:10.1016/1352-2310(96)00024-6.

HARGER, W. P., AREY, J. \& ATKINSON, R., 1992, The mutagenicity of HPLC-separated vapor-phase and particulate organics in ambient air. Atmos. Environ., 26A: 2463-2466. doi:10.1016/0960-1686(92)90378-X.

IARC, 1987, Overall evaluations of carcinogenicity an updating of IARC monographs, 1-42. IARC, Lyon. http://www.iarc.fr.

KADO, N. Y., GUIRGUIS, N., GUIRGUIS, C., FLESSEL, P., CHAN, R. C., CHANG, K. \& WESOLOWSKI, J. I., 1986, Mutagenicity of fine $(<2.5 \mu \mathrm{M})$ airborne particles: diurnal variation in community air determined by a Salmonella micro preincubation (microsuspension) procedure. Environ. Mutagen., 8: 53-66. doi:10.1002/em.2860080106.

KODELL, R. L., AHN, H., CHEN, J. J., SPRINGER, J. A., BARTON, C. N. \& HERTZBERG, R. C., 1995, Upper bound risk estimates for mixtures of carcinogens. Toxicology., 105: 199208. doi:10.1016/0300-483X(95)03213-Y.

LEWTAS, J. \& GALLAGHER, J., 1990, Complex mixtures of urban air pollutants: identification and comparative assessment of mutagenic and tumorigenic chemicals and emission sources, IARC Sci. Publ., 252-260.

MARON, D. M. \& AMES, B. N., 1983, Revised methods for the Salmonella mutagenicity test. Mutat Res., 11: 173-215. doi:10.1016/0165-1161(83)90010-9.

MIGUEL, A. G., DAISEY, J. M. \& SOUSA, J. A., 1990, Comparative study of the mutagenic and genotoxic activity associated with inhalable particulate matter in Rio de Janeiro air. Environ. Mol. Mutagen., 15: 36-43. doi: 10.1002/em.2850150106.

MYERS, L., ADAMS, N., KIER, L., RAO, T., SHAW, B. \& WILLIAMS, L., 1991, Microcomputer software for data management and statistical analyses of the Ames/Salmonella test. In: D. Krewski (ed), Statistical methods in toxicological research. Gordon And Brech, New York, p. 265-279.

PHOUSONGPHOUANG, P. \& AREY, J., 2003a, Rate constant for the gas-phase reactions of a series of alkylnaphthalenes with the nitrate radical. Environ. Sci. Technol., 37: 308-313. doi: 10.1021/ es026015+.

PHOUSONGPHOUANG, P. \&AREY, J., 2003b, Rate constant for the photolysis of the nitronaphthalenes and methylnitronaphthalenes. J. Photochem. Photobiol A: Chem., 157: 301-309. doi:10.1016/ S1010-6030(03)00072-8.

REINEKE, N., BESTER, K., HÜHNERFUSS, H., JASTORFF, B. \& WEIGEL, S., 2002, Bioassay-directed chemical analysis of River Elbe surface water including large volume extractions and high performance fractionation. Chemosphere, 47: 717-723. doi:10.1016/S0045-6535(01)00340-X.

REISEN, F., WHEELER, S. \& AREY, J., 2003, Methyl- and dimethyl-/ethyl-nitronaphthalenes measured in ambient air in Southern California. Atmos. Environ., 37: 3653-3657. doi:10.1016/S1352-2310(03)00469-2.

ROSENKRANZ, H. S., 1996, Mutagenic nitroarenes, diesel emissions, particulate-induced mutations and cancer: an assay on cancer-causation by a moving target. Mutat. Res., 367: 65-72. doi:10.1016/0165-1218(95)00066-6.

SATO, M. I. Z., VALENT, G. U., COIMBRÃO, C. A., COELHO, M. C. L. S., SANCHEZ, P. S., ALONSO, C. D. \& MARTINS, M. T., 1995, Mutagenicity of airborne particulate organic material from urban and industrial areas of São Paulo, Brazil. Mutat. Res., 335: 317-330. doi:10.1016/0165-1161(95)00035-6.

TOKIWA, H., 1980, Mutagenic, chemical assay of extracts of airborne particulates. Mutat. Res., 77: 99-108. doi:10.1016/01651218(80)90126-3.

UMBUZEIRO, G. A. \& VARGAS, V. M. F., 2003, Teste de mutagenicidade com Salmonella typhimurium (Teste de Ames) como indicador de carcinogenicidade em potencial para mamíferos. In: L. R. Ribeiro, D. M. F. Salvadori \& E. K. Marques (orgs), Mutagênese Ambiental. ULBRA, Canoas, 356p.

USEPA, 1986, Guidelines for cancer risk assessment, Fed. Reg. 51: 33992-34003.

USEPA, 2003, Draft final guidelines for cancer risk assessment (external review draft, February 2003), US Environmental Protection Agency, Risk Assessment Forum, Washington, DC.

VARGAS, V. M. F., 2003, Mutagenic activity as a parameter to assess ambient air quality for protection of the environment and human health. Mutat. Res., 544: 313-319. doi:10.1016/j. mrrev.2003.06.020.

VARGAS, V. M. F., HORN, R. C., GUIDOBONO, R. R., MITTELSTAEDT, A. B. N. \& AZEVEDO, I. M. G., 1998, Mutagenic activity of airborne particulate matter from urban areas of Porto Alegre, Brazil. Genet. Molec. Biol., 21: 1-7. doi: 10.1590/S1415-47571998000200013.

VARGAS, V. M. F., MOTTA, V. E. P. \& HENRIQUES, J. A. P., 1993, Mutagenic activity detected by the Ames test in river water under the influence of petrochemical industries. Mutat. Res., 319: 31-45. doi:10.1016/0165-1218(93)90028-C.

WATANABE, M., ISHIDATE Jr., M. \& NOHMI, T., 1989, A sensitive method for the detection of mutagenic nitroarenes: construction of nitroreductase-overpruducing derivatives of $S$. typhimurium strains TA98 and TA100. Mutat. Res., 216: 211220. doi:10.1016/0165-1161(89)90007-1.

WATANABE, M., ISHIDATE Jr., M. \& NOHMI, T., 1990, Sensitive method for detection of mutagenic nitroarenes and aromatic amines: new derivatives of Salmonella typhimurium tester strains possessing elevated O- acetyl-transferase levels. Mutat. Res., 234: 337-348. doi:10.1016/0165-1161(90)90044-O. 